\title{
A Data-Based Approach for Modeling and Analysis of Vehicle Collision by LPV-ARMAX Models
}

\author{
Qiugang Lu, Hamid Reza Karimi, and Kjell Gunnar Robbersmyr \\ Department of Engineering, Faculty of Engineering and Science, University of Agder, P.O. Box 509, 4898 Grimstad, Norway \\ Correspondence should be addressed to Hamid Reza Karimi; hamid.r.karimi@uia.no
}

Received 30 March 2013; Revised 21 May 2013; Accepted 21 May 2013

Academic Editor: Zhiwei Gao

Copyright (C) 2013 Qiugang Lu et al. This is an open access article distributed under the Creative Commons Attribution License, which permits unrestricted use, distribution, and reproduction in any medium, provided the original work is properly cited.

\begin{abstract}
Vehicle crash test is considered to be the most direct and common approach to assess the vehicle crashworthiness. However, it suffers from the drawbacks of high experiment cost and huge time consumption. Therefore, the establishment of a mathematical model of vehicle crash which can simplify the analysis process is significantly attractive. In this paper, we present the application of LPV-ARMAX model to simulate the car-to-pole collision with different initial impact velocities. The parameters of the LPVARMAX are assumed to have dependence on the initial impact velocities. Instead of establishing a set of LTI models for vehicle crashes with various impact velocities, the LPV-ARMAX model is comparatively simple and applicable to predict the responses of new collision situations different from the ones used for identification. Finally, the comparison between the predicted response and the real test data is conducted, which shows the high fidelity of the LPV-ARMAX model.
\end{abstract}

\section{Introduction}

In the past few decades, as one of the major concerns in the automotive industry, the vehicle crashworthiness has been attracting exceptional attention all over the world. Before appearing on roads, each car must undergo a series of crash tests to be verified whether they satisfy the safety requirements and conform to safety standards set by the road safety organizations or rate programs such as Euro NCAP or National Highway Traffic Safety Administrations (NHTSA). In terms of the crash tests, they are exceptionally costly and complicated due to the need of appropriate facilities, qualified staff, and data-acquisition system. Moreover, the proper arrangement of measuring devices (e.g., accelerometers and cameras used to record the crash event) or precise positioning of the research centers has also added the complexity of such crash tests. Thus it would be highly attractive if the overall car performance can be predicted and assessed without the need to execute numerous full-scale crash experiments, which renders the proposition of a mathematical models with accurate input-output behavior as the real tests to be of great interest.

Recently we can distinguish two main approaches of vehicle crash modeling: finite element method (FEM) simulations and lumped parameter modeling (LPM). FEM is considered as the most thorough computational tool with detailed insight into the vehicle crash modeling and thus has been widely employed; refer to [1-4] and the references therein. It offers high accuracy of results and makes it possible to describe large number of details such as connections and interactions between particular bodies, their deformation rate, or material properties. The major disadvantages of this method include the high computational demand of powerful hardware and the purchase of software used to perform the numerical simulation. The second approach frequently used in vehicle crash is an analytical method of formulating a model called lumped parameter modeling (LPM). It consists of formulating equations of motion of spring-damper-mass systems and solving them so as to precisely determine the model's response. However, the drawback of this method is that it can perform well only when used for a given set of collisions. With respect to this shortcoming, several methodologies such as artificial neural networks and wavelet transforms are utilized to make it possible to simulate various crash scenarios; see [5].

On the other hand, for the modeling of complex systems, the model identification based on the data training or estimation can be an alternative to the traditional modeling by using physical laws $[6,7]$. Up to now, the data-based modeling for 


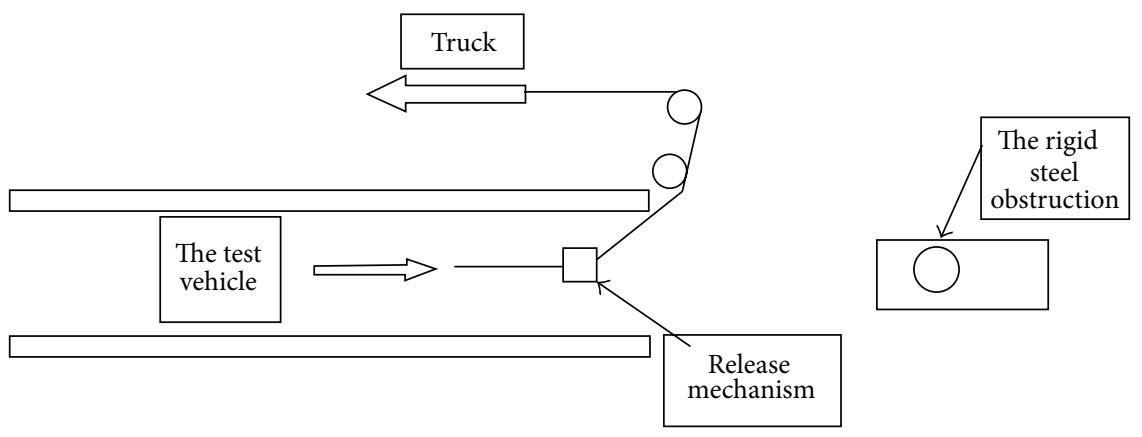

Figure 1: Scheme of crash test.
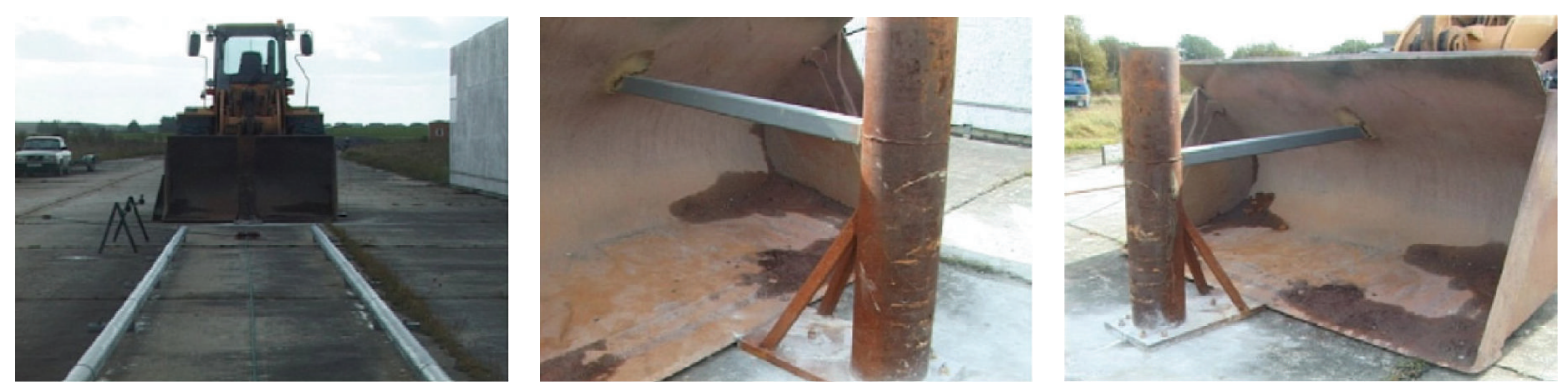

Figure 2: Obstruction.

the vehicle crashworthiness has been extensively investigated. It has been well proven that the applications of autoregressive models like nonlinear autoregressive (NAR), nonlinear autoregressive with exogenous input (NARX), or nonlinear autoregressive moving average with exogenous input (NARMAX) are capable of predicting the time-series data with high fidelity. Up to now, a wide range of areas in the applications of autoregressive models have been well explored. In [8], the ARMA model of edge-localized modes (ELM) time series has been established, which can decompose the time series into deterministic and noise components. A hybrid of NARX and ARMA models was employed to predict the longterm machine state based on vibration data in [9]. NARX models have been successfully applied to black box modeling of the gas turbine operating in isolated and nonisolated modes in [10]. The prediction performance assessment of recurrent NARX model was investigated in [11], in which the effects of the changing network's architecture on the quality of predictions are verified. Apart from the technical applications, the regressive models have also been widely employed as an advantageous methodology in other areas of research, such as stock market [12], cardiovascular diseases prevention [13], and prediction of freshwater phytoplankton dynamics [14].

A significant contribution to data-based approach in modeling automobile crash was made in [15], where the autoregressive moving average (ARMA) model was used to estimate lumped parameters (i.e., stiffness, damping, and masses) of analytical models (differential equations). Those physical parameters are changeable in time, which thus makes it possible to simulate the vehicle frontal and side impacts with high level of accuracy. What is more, the

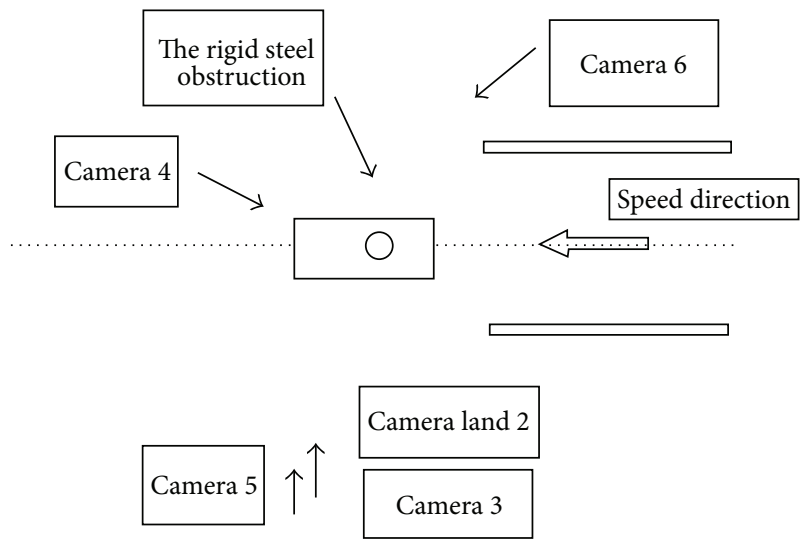

FIgURE 3: Cameras layout.

neural networks have been successfully used to estimate the parameters of vehicle crash models. Specifically, the values of the spring stiffness and damping coefficients for the LPM were obtained through the radial basis artificial neural network (RBFNN) in [16]. Artificial neural networks (ANNs) were also implemented in [17] to predict the crash severity and occupants injury. Other up-to-date technologies currently utilized in vehicle crashworthiness modeling include wavelets, fuzzy logic, and intelligent approach; refer to [1820].

Recently, the research scope of crashworthiness has been focused on defining a dynamic vehicle crash with changing parameters according to the changeable input such as initial impact velocity [21, 22]. In particular, in [21], a nonlinear occupant model is established and a scheduling variable is 

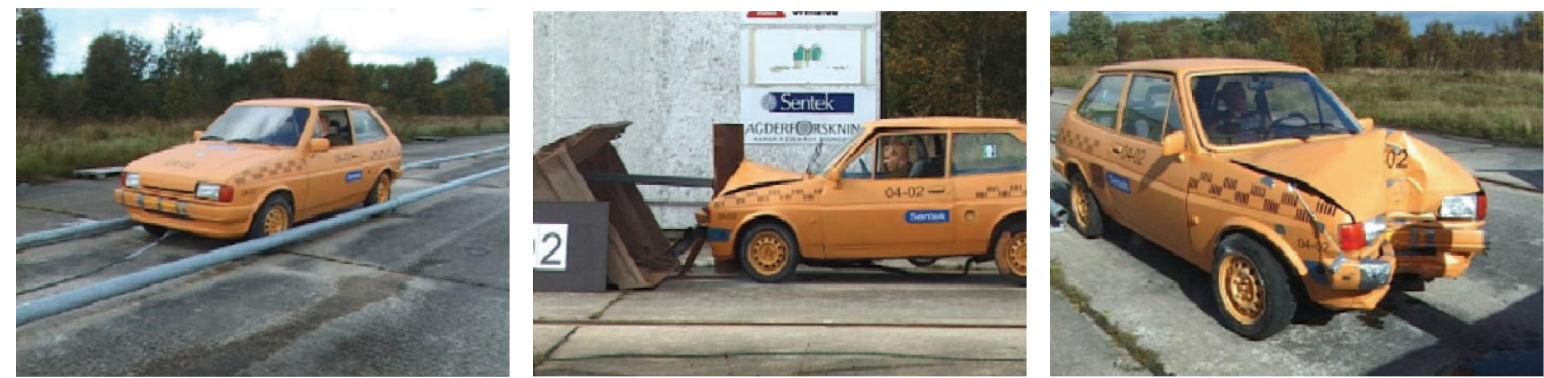

FIgURE 4: Car's deformation
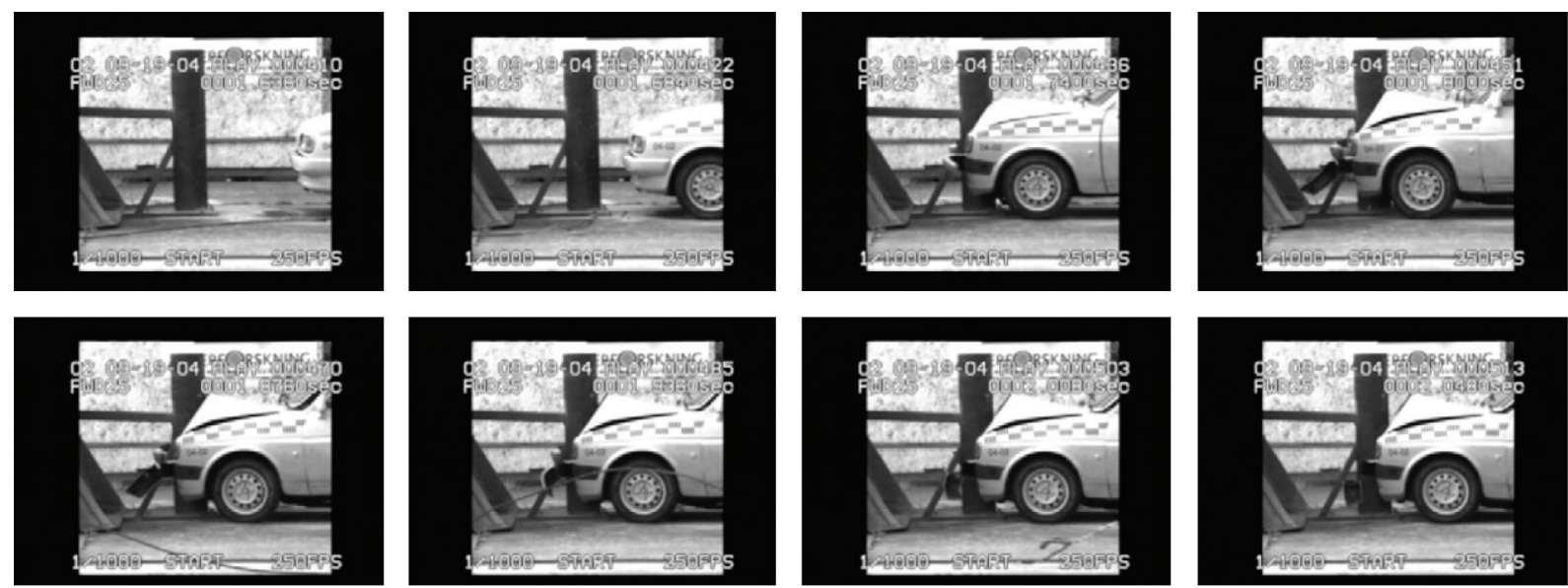

FIGURE 5: Steps of the experiment recorded by the high-speed camera.

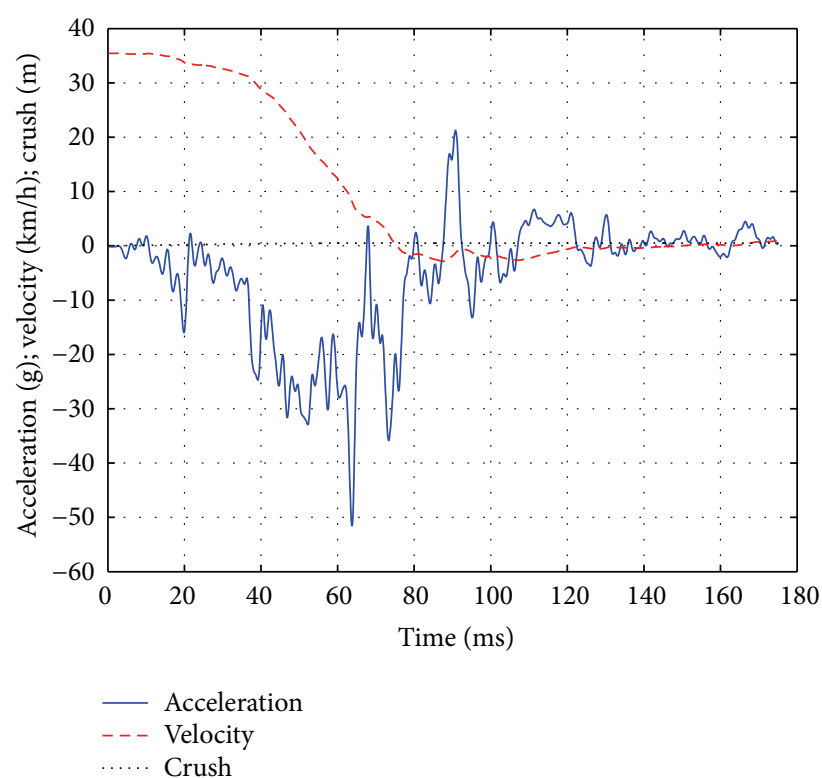

FIgURE 6: Vehicle kinematics with $\bar{v}=35 \mathrm{~km} / \mathrm{h}$.

defined to formulate the linear parameter-varying (LPV) model. Inspired by this work, in this paper, we aim to establish the LPV-ARMAX models for the vehicle crash, and the scheduling parameter is selected as the initial impact velocity. This LPV model consists of a set of ARMAX models in which

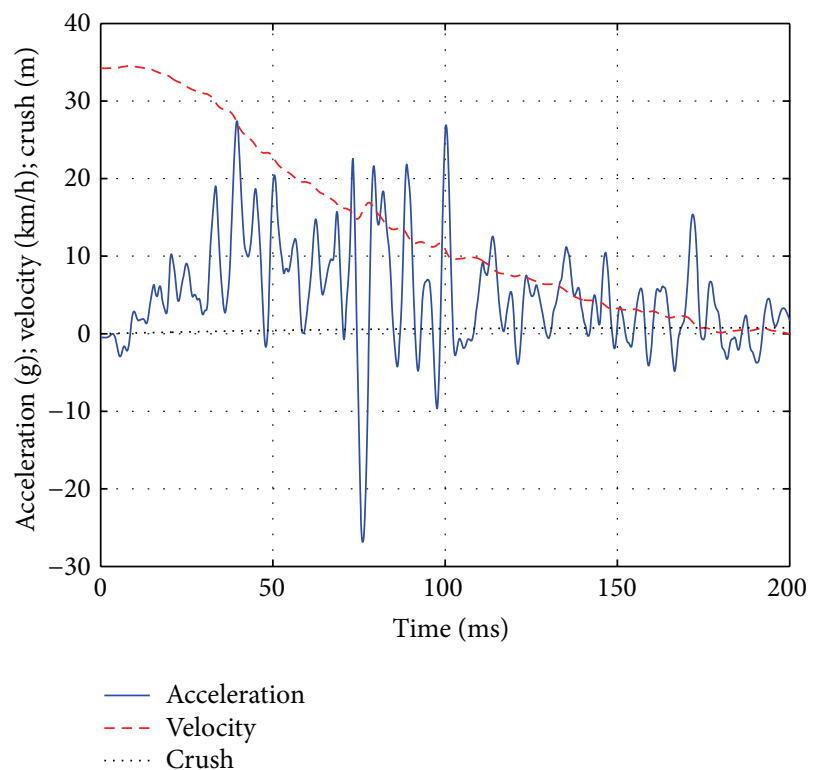

Figure 7: Vehicle kinematics with $\bar{v}=35.57 \mathrm{~km} / \mathrm{h}$.

the parameters are not constant but are functions of the impact velocity that can be regarded as an input determining the "operating condition" of the model [23]. So instead of generating a large database of ARMAX models for every possible impact velocity, we can calculate a single model that is function of impact velocity, which is far more attractive. 


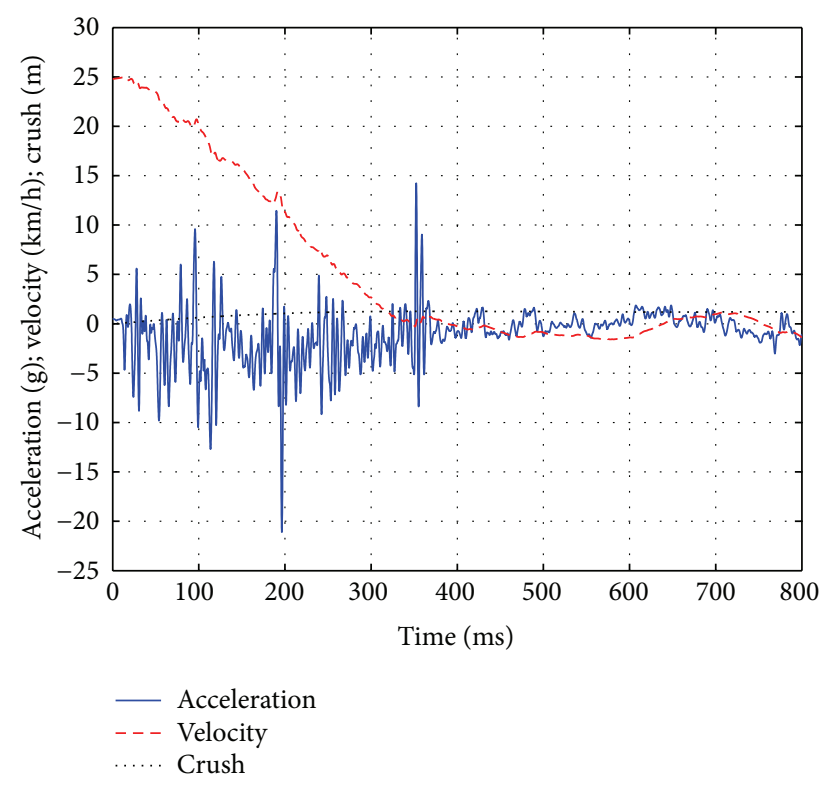

FIGURE 8: Vehicle kinematics with $\bar{v}=24.63 \mathrm{~km} / \mathrm{h}$.

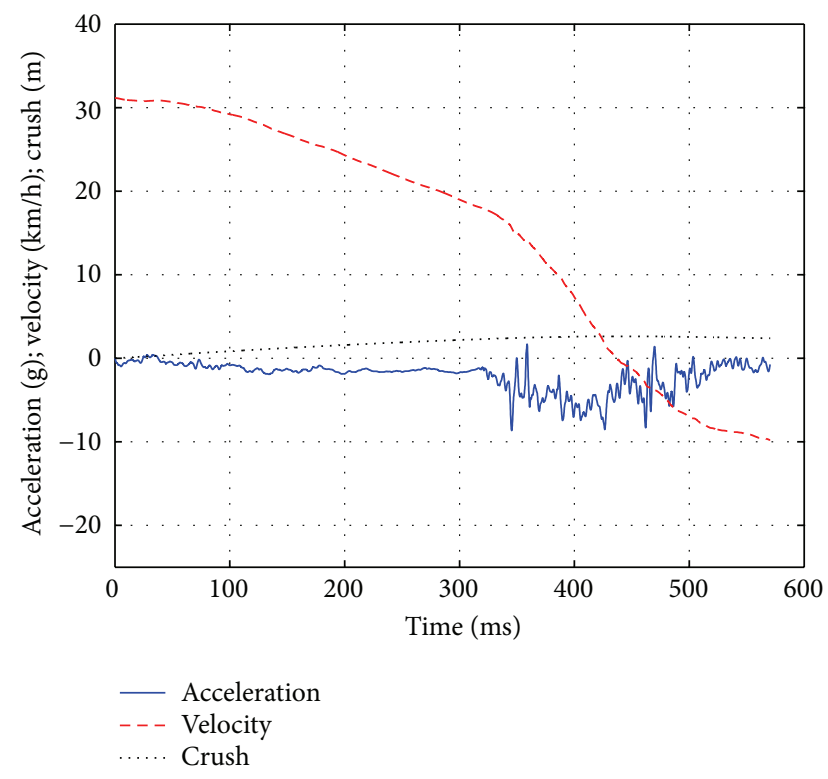

FIGURE 9: Vehicle kinematics with $\bar{v}=31.35 \mathrm{~km} / \mathrm{h}$.

The structure of this paper is organized as follows. The detailed introduction about the full-scale vehicle-to-pole collision is presented in Section 2. In Section 3, the notation and main ideas of this approach are developed. Section 4 illustrates the application of LPV-ARMAX-based time series analysis to the modeling of vehicle crash, and simulation results are analyzed and compared. Finally, the conclusion is given in Section 5.

\section{Crash Test Description}

In this section we present car-to-pole collisions with different initial impact velocities so as to test if the model proposed is capable of representing different crash scenarios.
The car tests were subjected to impact with a vertical and rigid cylinder. During the tests, the acceleration was measured in three directions, that is, longitudinal, lateral, and vertical. The yaw rate from the car's center of gravity was also recorded. The acceleration field was 100 meters long with two anchored parallel pipelines. The pipelines have a clearance of $5 \mathrm{~mm}$ to the front wheel tires. The force to accelerate the test car was generated using a truck and a tackle. The release mechanism was placed $2 \mathrm{~m}$ before the end of the pipelines, and the distance from there to the test item was $6.5 \mathrm{~m}$. The car was steered using the pipelines that were bolted to the concrete runaway. The experimental scheme is shown in Figure 1.

2.1. Description of the Car and Pole. As illustrated in Figure 2, the pole was constructed with two components: a baseplate and a pipe. The baseplate had dimensions $740 \times 410 \times 25 \mathrm{~mm}$. The pipe had a length of $1290 \mathrm{~mm}$ and overall diameter equal to $275 \mathrm{~mm}$. The pipe was filled with concrete and mounted on a concrete foundation with five bolts. These bolts connected the concrete foundation with the baseplate of the obstruction which was fixed to the shovel of a bulldozer. The initial velocities of the cars are $35 \mathrm{~km} / \mathrm{s}, 35.57 \mathrm{~km} / \mathrm{h}, 24.63 \mathrm{~km} / \mathrm{h}$, and $31.35 \mathrm{~km} / \mathrm{h}$.

2.2. Instrumentation. During the test, the acceleration at the center of gravity in three dimensions ( $x$-longitudinal, $y$ lateral, and $z$-vertical) was recorded. The car speed before the collision was measured. The yaw rate was also measured with a gyrometer. Using normal speed and high-speed video cameras, the behavior of the safety barrier and the test car during the collision was recorded; see Figure 3.

A 3-D accelerometer was mounted on a steel bracket close to the car's center of gravity, and it was fastened by screws to the car's chassis. Data from the sensor was fed to an eight-channel data logger and subsequently sampled with a frequency of $10 \mathrm{kHz}$. The memory was able to store $6.5 \mathrm{~s}$ of data per channel. The velocity of the car was checked by an inductive monitor. It was directed towards a perforated disc mounted on a wheel on the right side of the test car. Figure 4 shows the car before, during, and after the collision. The steps of the experiment recorded by the high-speed camera are illustrated in Figure 5.

2.3. Analysis of Crash Pulse. All the tests are central impact; thus, as already mentioned only the pulse recorded in the longitudinal direction ( $x$-axis) is analyzed. By integrating car's acceleration

$$
\begin{aligned}
& v(t)=\int_{t_{0}}^{t} a(\tau) \mathrm{d} \tau, \\
& x(t)=\int_{t_{0}}^{t} v(\tau) \mathrm{d} \tau,
\end{aligned}
$$

we can obtain plots of velocity $v(t)$ and crush (or displacement) $x(t)$ for different initial impact velocities $\bar{v}$; see Figures $6,7,8$, and 9. Take the vehicles kinematics with $\bar{v}=$ $35 \mathrm{~km} / \mathrm{h}$ (Figure 6) as an example. At the time when the 


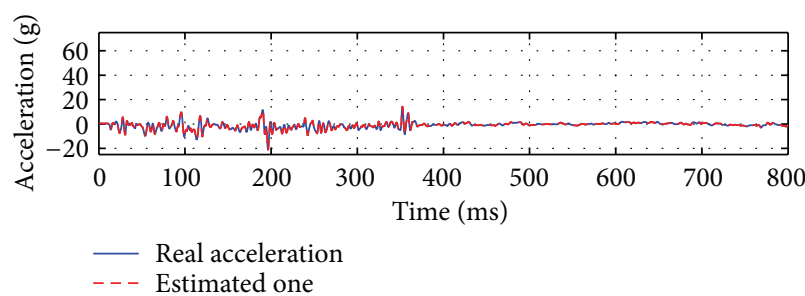

(a)

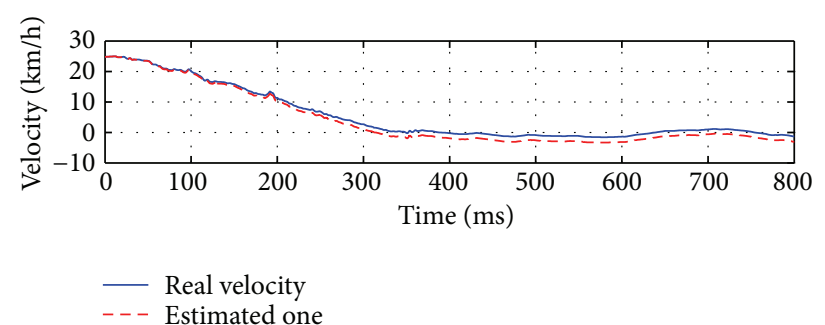

(b)

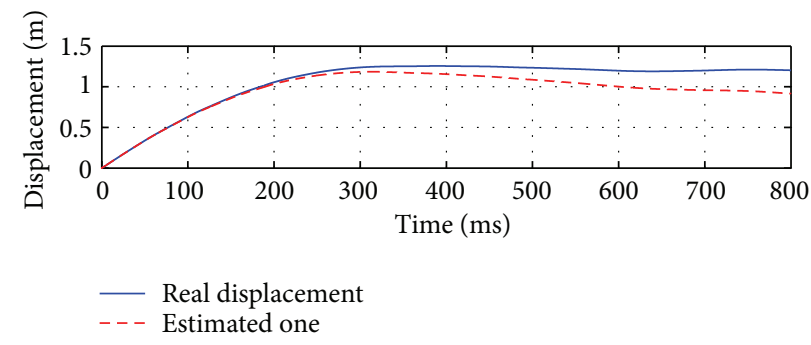

(c)

FIgURE 10: ARMAX simulation results for vehicle with $\bar{v}=24.63 \mathrm{~km} / \mathrm{h}$.

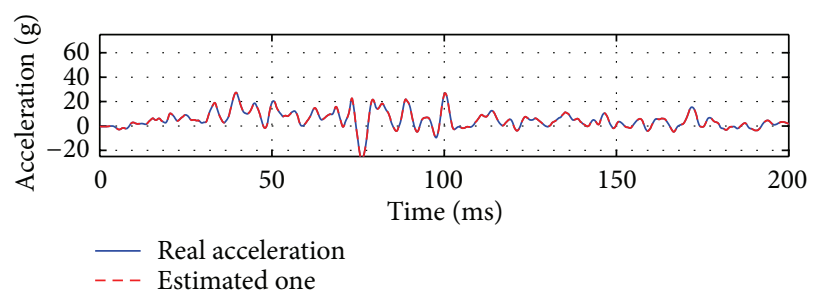

(a)

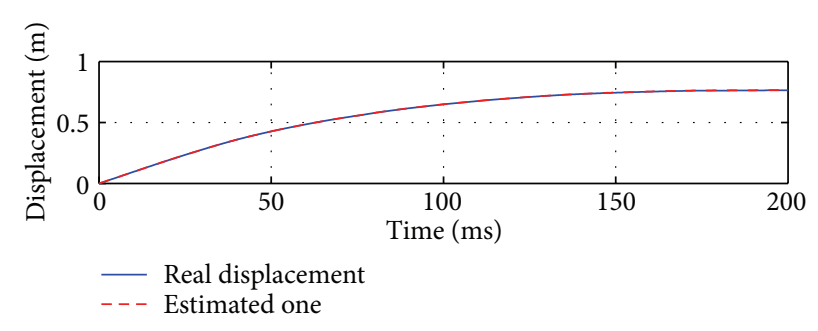

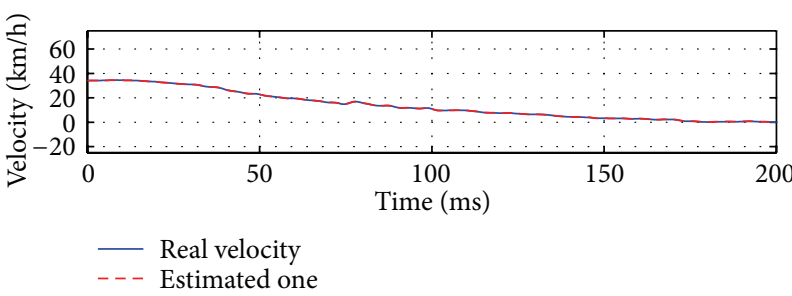

(b)

(c)

FIGURE 11: ARMAX simulation results for vehicle with $\bar{v}=35.57 \mathrm{~km} / \mathrm{h}$.

relative approach velocity is zero, the maximum dynamic crush occurs. The relative velocity in the rebound phase then increases negatively up to the final separation (or rebound) velocity, at which time a vehicle rebounds from an obstacle. The contact duration of the two masses includes both contact time in deformation and restitution phases. When the relative acceleration becomes zero and relative separation velocity reaches its maximum recoverable value, we have the separation of the two masses.

\section{Mathematical Modeling}

There are two types of mathematical modelings of real world systems that are commonly used [24]. The first is mathematical approach, in which the dynamics of a system is derived from the fundamental law of physics, for example, Newton's Laws or conservation principle. The secondary approach is the system identification. It is a process to select the model's parameters so that the model's behavior can fit the experimental data. In this paper, we adopt the second approach.

3.1. ARMAX Model Description. Analysis of the autoregressive model with moving average exogenous input (ARMAX) was done according to [24]. The ARMAX model can be defined as

$$
y(t)+a_{1} y(t-1)+\cdots+a_{n_{a}} y\left(t-n_{a}\right)
$$




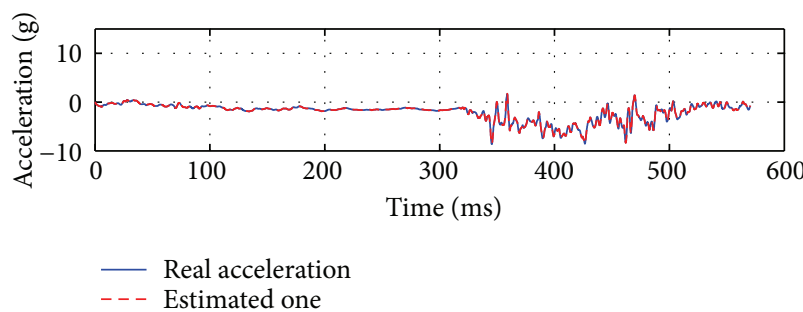

(a)

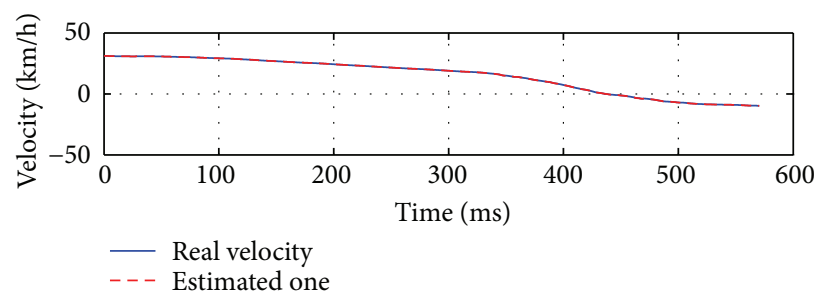

(b)

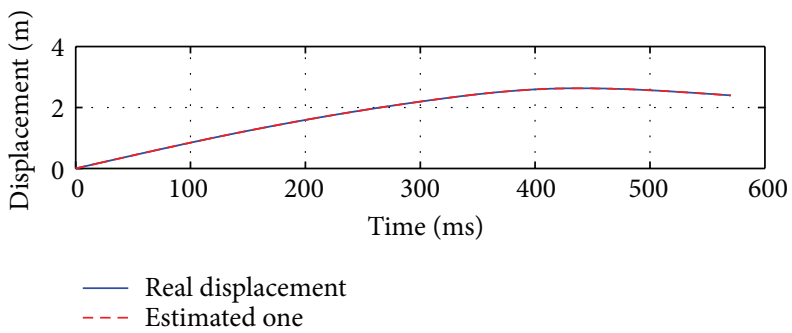

(c)

FIGURE 12: ARMAX simulation results for vehicle with $\bar{v}=31.35 \mathrm{~km} / \mathrm{h}$.

$$
\begin{gathered}
=b_{1} u\left(t-n_{k}\right)+\cdots+b_{n_{b}} u\left(t-n_{k}-n_{b}+1\right) \\
+c_{1} e(t-1)+\cdots+c_{n_{c}} e\left(t-n_{c}\right)+e(t),
\end{gathered}
$$

where $t$ is the time, $y(t)$ is system's output, and $a_{1}, \ldots, a_{n_{a}}$, $b_{1}, \ldots, b_{n_{b}}, c_{1}, c_{2}, \ldots, c_{n_{c}}$ are model's parameters. $n_{a}, n_{b}$, and $n_{c}$ are, respectively, the number of model's poles, model's zeros +1 , and parameters in $c$ vector. $n_{k}$ is the order of input delay. $y(t-1), \ldots, y\left(t-n_{a}\right)$ are system's outputs in previous moments and $u(t-1), \ldots, u\left(t-n_{b}\right)$ are system's inputs in previous moments. $e(t-1), \ldots, e\left(t-n_{c}\right)$ are white noise.

The ARMAX model can be simplified as

$$
A\left(q^{-1}\right) y(t)=B\left(q^{-1}\right) u\left(t-n_{k}\right)+C\left(q^{-1}\right) e(t),
$$

where

$$
\begin{aligned}
& A\left(q^{-1}\right)=1+a_{1} q^{-1}+a_{2} q^{-2}+\cdots+a_{n_{a}} q^{-n_{a}}, \\
& B\left(q^{-1}\right)=b_{1}+b_{2} q^{-1}+\cdots+b_{n_{b}} q^{-n_{b}}, \\
& C\left(q^{-1}\right)=c_{1}+c_{2} q^{-1}+\cdots+c_{n_{c}} q^{-n_{c}} .
\end{aligned}
$$

3.2. LPV-ARMAX Model Establishment. As stated in Section 1, the ARMAX models of vehicle crash in most literature are assumed to be linear time invariant (LTI). So for collisions under different initial conditions (e.g., initial impact velocities and vehicle masses), we have to extract different ARMAX models accordingly, which suffer from serious limitation especially when the number of LTI-ARMAX models is huge. Thus it is quite attractive if we can establish one LPV-ARMAX model whose parameters are functions of initial condition variables and are applicable to collision under a wide range of initial situations. Consider the parameters in the LPV-ARMAX models of the vehicle crash which are dependent on the initial impact velocity $\bar{v}$. What we are interested in is to find the dependence relationship and predict parameters and then the output of the LPV-ARMAX models for any initial impact velocity.

In the following, we will adopt a LPV-ARMAX model which can be described as

$$
A\left(q^{-1} ; \bar{v}\right) y(t ; v)=B\left(q^{-1} ; \bar{v}\right) u\left(t-n_{k}\right)+C\left(q^{-1} ; \bar{v}\right) e(k),
$$

where

$$
\begin{aligned}
& A\left(q^{-1} ; \bar{v}\right)=1+a_{1}(\bar{v}) q^{-1}+a_{2}(\bar{v}) q^{-2}+\cdots+a_{n_{a}}(\bar{v}) q^{-n_{a}} \\
& B\left(q^{-1} ; \bar{v}\right)=b_{1}(\bar{v})+b_{2}(\bar{v}) q^{-1}+\cdots+b_{n_{b}}(\bar{v}) q^{-n_{b}} \\
& C\left(q^{-1} ; \bar{v}\right)=c_{1}(\bar{v})+c_{2}(\bar{v}) q^{-1}+\cdots+c_{n_{c}}(\bar{v}) q^{-n_{c}}
\end{aligned}
$$

Note that the parameters $a_{i}(\bar{v}), i=1,2, \ldots, n_{a}, b_{j}(\bar{v}), j=$ $1,2, \ldots, n_{b}$ and $c_{j}(\bar{v}), j=1,2, \ldots, n_{c}$ are continuous functions of the impact velocity $\bar{v}$. The specific dependence form can be selected as polynomials, radical basis functions, splines, or other linear combinations of given functions. We define this model as LPV-ARMAX $\left(n_{a}, n_{b}, n_{c}\right)$ for simplicity.

The following instructions specify the steps of such identification.

(1) Suppose that we have a set of groups of vehicle crash data, all of them are in the type of pole collision but under different impact velocities $\left\{\bar{v}_{1}, \bar{v}_{2}, \ldots, \bar{v}_{n}\right\}$. For any $\bar{v}_{j}, j=1,2, \ldots, n$, a set of time-series data is collected. From this data set, an LTI-ARMAX model with the same structure of the LPV-ARMAX model is identified. Thus, $n$ distinct LTI-ARMAX models are obtained, each for $\bar{v}_{j}, j=1,2, \ldots, n$. And we can get the $j$ th ARMAX model parameters $a_{i}\left(\bar{v}_{j}\right)$, $i=1,2, \ldots, n_{a}, b_{i}\left(\bar{v}_{j}\right), i=1,2, \ldots, n_{b}$ and $c_{i}\left(\bar{v}_{j}\right)$, $i=1,2, \ldots, n_{c}$. 


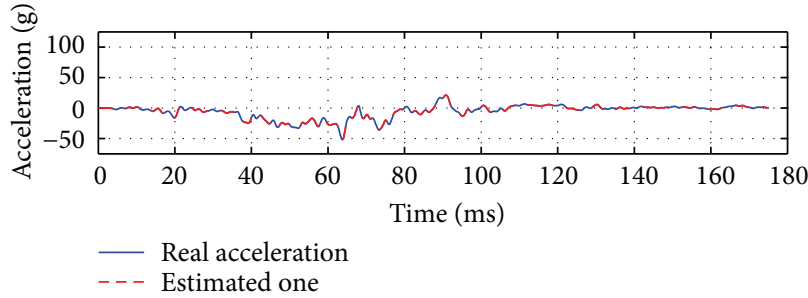

(a)

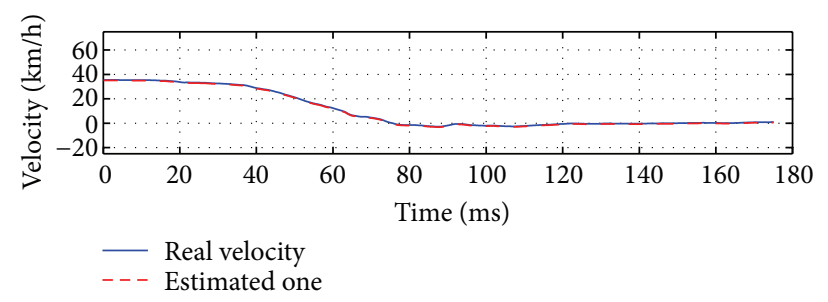

(b)

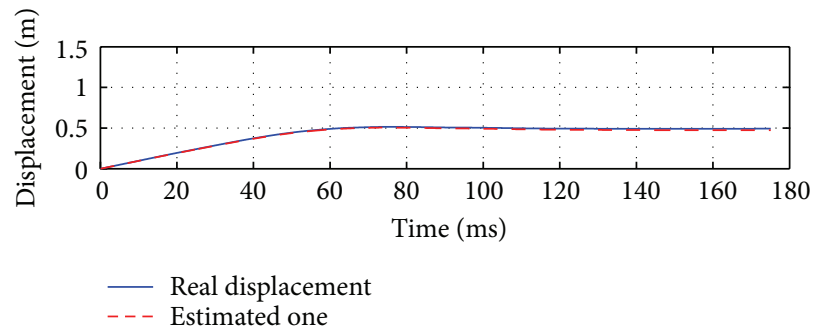

(c)

FIGURE 13: LPV-ARMAX perdition for vehicle with $\bar{v}=35 \mathrm{~km} / \mathrm{h}$.

(2) The $n$ different values of the same parameter $\left(a_{i}\left(\bar{v}_{j}\right)\right.$, $\left.b_{i}\left(\bar{v}_{j}\right), c_{i}\left(\bar{v}_{j}\right)\right)$ in the $n$ distinct LTI-ARMAX models obtained in step (1) can then be used to identify the dependence functions $\left(a_{i}(\bar{v}), b_{i}(\bar{v}), c_{i}\left(\bar{v}_{j}\right)\right)$. These dependence functions may be, for instance, polynomials or splines, which can result in the LPV parameters by interpolating the parameters of the $n$ LTI-ARMAX models.

From the resulting LPV model, the output $y(t ; \bar{v})$ then can be obtained for any initial impact velocity $\bar{v}$, even different from those utilized in the identification process.

When the functions $a_{i}(\bar{v}), i=1,2, \ldots, n_{a}, b_{i}(\bar{v}), i=$ $1,2, \ldots, n_{b}$ and $c_{i}(\bar{v}), i=1,2, \ldots, n_{c}$, are described using generalized polynomials such as $B$ splines, that is,

$$
\begin{aligned}
& a_{i}(\bar{v})=\sum_{j=1}^{n_{s}} \alpha_{i, j} \varphi_{j}(\bar{v}), \\
& b_{i}(\bar{v})=\sum_{j=1}^{n_{s}} \beta_{i, j} \varphi_{j}(\bar{v}), \\
& c_{i}(\bar{v})=\sum_{j=1}^{n_{s}} \gamma_{i, j} \varphi_{j}(\bar{v}),
\end{aligned}
$$

where $\varphi_{j}(\bar{v}), j=1,2, \ldots, n_{s}$ are linear independent continuous functions, the parameter-dependent ARX can be written as

$$
\begin{aligned}
y(t ; \bar{v})= & \sum_{i=1}^{n_{a}} \sum_{j=1}^{n_{s}} \alpha_{i, j} \varphi_{j}(v) y(t-i ; \bar{v}) \\
& +\sum_{i=0}^{n_{b}} \sum_{j=1}^{n_{s}} \beta_{i, j} \varphi_{j}(v) u(t-i) \\
& +\sum_{i=0}^{n_{c}} \sum_{j=1}^{n_{s}} \gamma_{i, j} \varphi_{j}(v) e(t-i),
\end{aligned}
$$

where $\alpha_{i, j}, i=1,2, \ldots, n_{a}, j=1,2, \ldots, n_{s}, \beta_{i, j}, i=1,2, \ldots, n_{b}$, $j=1,2, \ldots, n_{s}$, and $\gamma_{i, j}, i=1,2, \ldots, n_{c}, j=1,2, \ldots, n_{s}$ are the parameters to be estimated.

It should be noted that the LPV-ARMAX model is linear with respect to $\alpha_{i, j}, \beta_{i, j}$, and $\gamma_{i, j}$. Assuming the error $e(t)$ to be Gaussian, it is possible to estimate the parameters using the techniques in [25].

Remark 1. It should be noted that the recorded acceleration signal in real car crash test is actually time-series data. Thus in the established LPV-ARMAX model $(5), B\left(q^{-1} ; \bar{v}\right)=0$ for the vehicle crash since there exists no input (as will be shown in the simulation part), and the output $y(t ; \bar{v})$ of (5) corresponds to the acceleration signal in the vehicle crash test.

Remark 2. In the previous literature, the most commonly used method is to establish an ARMAX model based on a group of vehicle crash data obtained under one specific impact velocity, which may not be applicable to other situations with different impact velocities. Thus it is desirable to know the models in the whole relevant volume of initial velocity instead of one (or several) specific values. Moreover, since the impact velocity can be arbitrarily valued in practice, the corresponding local model can be obtained easily with aid of the LPV-ARMAX model once we know the specific initial velocity, which is the advantage of our approach.

In the next section, we will present the simulation of vehicle crash to test the efficiency proposed in this paper. In the simulation, the error noise is Gaussian, and the lumped LTI-ARMAX models are identified via the MATLAB Identification Toolbox.

\section{Simulation Results}

In this section, the parameters of the LPV-ARMAX are estimated. We have four data sets corresponding to four initial impact velocities at our disposal, of which three data sets will be used to obtain the LPV-ARMAX model while 


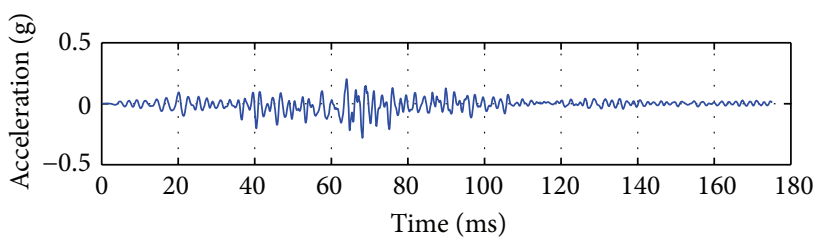

(a)

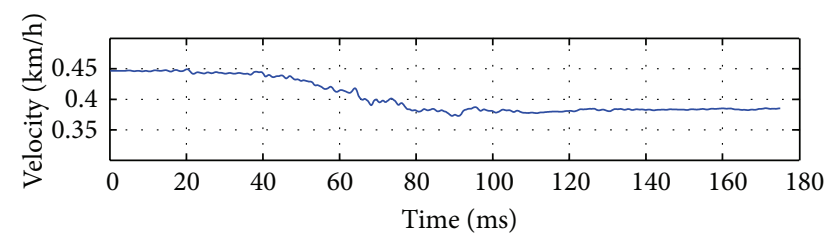

(b)

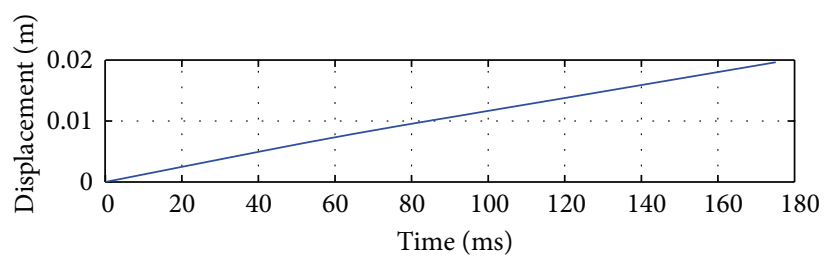

(c)

FIGURE 14: Simulation error by LPV-ARMAX model for vehicle with $\bar{v}=35 \mathrm{~km} / \mathrm{h}$.

the rest data set will be used for the purpose of verification. If one data set under a specific initial impact velocity $\bar{v}=$ $\bar{v}^{*}$ is considered, the recorded acceleration signal can be approximated with a time-invariant ARMAX model. The derived LTI model can account well for the initial impact velocity at $\bar{v}^{*}$. However, the obtained results do not give any information about initial impact velocity in any other section different from $\bar{v}^{*}$. Therefore, for the sake of prediction, the use of this LTI model is inadequate.

With this in mind, in order to represent with a simplified model of the vehicle crash that can be applicable to the collisions with different initial impact velocity, the LPV-ARMAX model should be well constructed. It is assumed that the time series data of acceleration during the collision can be well represented by a LPV-ARMAX $(2,0,2)$ (note that for time series data, $\left.n_{b}=0\right)$ model whose parameters $a_{1}(\bar{v}), a_{2}(\bar{v})$, $c_{1}(\bar{v})$, and $c_{2}(\bar{v})$ are functions of the initial impact velocity $\bar{v}$. The functions relating the parameters to impact velocity $\bar{v}$ are assumed to be cubic splines obtained by the interpolation of the parameters of three LTI-ARMAX $(2,0,2)$ models at three impact velocities. In this simulation, the four data sets selected to get the LPV-ARMAX model are those with impact velocities $\bar{v}=\{24.63 \mathrm{~km} / \mathrm{h}, 31.35 \mathrm{~km} / \mathrm{h}, 35.57 \mathrm{~km} / \mathrm{h}\}$. The obtained three LTI-ARMAX models and their simulation results are illustrated in Table 1 and Figures 10, 11, and 12, respectively.

From Table 1, we can get the resulting LPV-ARMAX $(2,0,2)$ model. Then for the vehicle crash with $\bar{v}=35 \mathrm{~km} / \mathrm{h}$, its LTI-ARMAX model can be obtained by interpolation as shown below:

$$
\begin{aligned}
& A(q)=1-1.9840 q^{-1}+0.9879 q^{-2} \\
& C(q)=1+1.1170 q^{-1}+0.6581 q^{-2} .
\end{aligned}
$$

With (9), the prediction of acceleration under the other impact velocity $\bar{v}=35 \mathrm{~km} / \mathrm{h}$ that is not used for identification is conducted. Figure 13 shows the simulation of the identified LPV model at impact velocity $\bar{v}=35 \mathrm{~km} / \mathrm{h}$, together with the integrated velocity and displacement. The acceleration data series obtained in real test are also illustrated in Figure 13.
TABLE 1: Parameters of ARMAX models for different impact velocities.

\begin{tabular}{lc}
\hline Impact velocity $\bar{v}$ & Parameters of corresponding ARMAX models \\
\hline $\bar{v}=24.63 \mathrm{~km} / \mathrm{h}$ & $A(q)=1-1.990 q^{-1}+0.9956 q^{-2}$ \\
& $C(q)=1+1.992 q^{-1}+0.992 q^{-2}$ \\
$\bar{v}=31.35 \mathrm{~km} / \mathrm{h}$ & $A(q)=1-1.987 q^{-1}+0.9871 q^{-2}$ \\
& $C(q)=1+1.993 q^{-1}+0.9938 q^{-2}$ \\
$\bar{v}=35.57 \mathrm{~km} / \mathrm{h}$ & $A(q)=1-1.983 q^{-1}+0.9883 q^{-2}$ \\
& $C(q)=1+0.9916 q^{-1}+0.5794 q^{-2}$ \\
\hline
\end{tabular}

Figure 14 shows the error response between the estimation and origin of the signal. It can be observed that the approximation is quite good, and the errors coming from LPVARMAX $(2,0,2)$ are so small that they can be neglected.

The simulation results show that the established LPVARMAX $(2,0,2)$ model can not only reproduce the crash pulse signals which were utilized for the identification, but also predict the kinematic responses of crash pulses with new impact velocity which were not presented in the creation stage. Thus, it can be proved that the LPV-ARMAX model allows us to obtain the accurate estimations of vehicle's acceleration signal under different initial conditions and therefore the integration of acceleration which closely resembles the original ones.

\section{Conclusion}

In this paper, a new method to approximate the modeling of the vehicle's acceleration pulses under different impact velocities is investigated by using LPV approach. The LPV-ARMAX model allowing to approximate the behavior of vehicle crash with various initial conditions is investigated. Specifically, the parameters of the LPV-ARMAX model are assumed to be functions of the initial impact velocity which may produce significant influence on the acceleration pulse during the crash. By interpolating the LTI-ARMAX models obtained from the training data sets, the overall LPV-ARMAX model 
can be achieved which can predict accurately the kinematic responses of vehicle crashes with various impact velocities. Simulation results illustrate that the LPV-ARMAX model can exactly reproduce both the new vehicle crash scenarios and those used for training. Future works may include taking the masses of vehicles into account as well since the masses can affect the acceleration pulses during the collision to some extent. But this attempt may increase the complexity since it will result in the coupling of the parameters in the LPVARMAX identification process, and thus it remains further investigation.

\section{References}

[1] T. Belytschko, "On computational methods for crashworthiness," Computers and Structures, vol. 42, no. 2, pp. 271-279, 1992.

[2] Z. Moumni and F. Axisa, "Simplified modelling of vehicle frontal crashworthiness using a modal approach," International Journal of Crashworthiness, vol. 9, no. 3, pp. 285-297, 2004.

[3] M. Borovinšek, M. Vesenjak, M. Ulbin, and Z. Ren, "Simulation of crash tests for high containment levels of road safety barriers," Engineering Failure Analysis, vol. 14, no. 8, pp. 1711-1718, 2007.

[4] C. A. Soto, "Structural topology optimization for crashworthiness," International Journal of Crashworthiness, vol. 9, no. 3, pp. 277-283, 2004.

[5] H. R. Karimi, W. Pawlus, and K. G. Robbersmyr, "Signal reconstruction, modeling and simulation of a vehicle full-scale crash test based on Morlet wavelets," Neurocomputing, vol. 93, no. 15, pp. 88-99, 2012.

[6] Z. Gao, D. Kong, and C. Gao, "Modeling and control of complex dynamic systems: applied mathematical aspects," Journal of Applied Mathematics, vol. 2012, Article ID 869792, 5 pages, 2012.

[7] Z. Gao, X. Dai, T. Breikin, and H. Wang, "Novel parameter identification by using a high-gain observer with application to a gas turbine engine," IEEE Transactions on Industrial Informatics, vol. 4, no. 4, pp. 271-279, 2008.

[8] G. Zvejnieks, V. N. Kuzovkov, O. Dumbrajs et al., "Autoregressive moving average model for analyzing edge localized mode time series on Axially Symmetric Divertor Experiment (ASDEX) Upgrade tokamak," Physics of Plasmas, vol. 11, no. 12, article 10, pp. 5658-5667, 2004.

[9] H. T. Pham, V. T. Tran, and B. Yang, "A hybrid of nonlinear autoregressive model with exogenous input and autoregressive moving average model for long-term machine state forecasting," Expert Systems with Applications, vol. 37, no. 4, pp. 3310-3317, 2010.

[10] M. Basso, L. Giarré, S. Groppi, and G. Zappa, "NARX models of an industrial power plant gas turbine," IEEE Transactions on Control Systems Technology, vol. 13, no. 4, pp. 599-604, 2005.

[11] R. Zemouri, R. Gouriveau, and N. Zerhouni, "Defining and applying prediction performance metrics on a recurrent NARX time series model," Neurocomputing, vol. 73, no. 13-15, pp. 2506-2521, 2010.

[12] C. Cheng, T. Chen, and L. Wei, "A hybrid model based on rough sets theory and genetic algorithms for stock price forecasting," Information Sciences, vol. 180, no. 9, pp. 1610-1629, 2010.

[13] L. Faes, G. Nollo, and K. H. Chon, "Linear and nonlinear parametric model identification to assess granger causality in short-term cardiovascular interactions," in Proceedings of the Computers in Cardiology (CAR '08), pp. 793-796, Bologna, Italy, September 2008.
[14] K. Jeong, D. Kim, J. Jung, M. Kim, and G. Joo, "Non-linear autoregressive modelling by Temporal Recurrent Neural Networks for the prediction of freshwater phytoplankton dynamics," Ecological Modelling, vol. 211, no. 3-4, pp. 292-300, 2008.

[15] U. N. Gandhi and S. J. Hu, "Data-based approach in modeling automobile crash," International Journal of Impact Engineering, vol. 16, no. 1, pp. 95-118, 1995.

[16] W. Pawlus, J. E. Nielsen, H. R. Karimi, and K. G. Robbersmyr, "Comparative analysis of vehicle to pole collision models established using analytical methods and neural networks," in Proceedings of the 5th IET International Conference on System Safety, Manchester, UK, October 2010.

[17] J. McFadden, W. Yang, and S. R. Durrans, "Application of artificial neural networks to predict speeds on two-lane rural highways," Transportation Research Record, no. 1751, pp. 9-17, 2001.

[18] H. R. Karimi and K. G. Robbersmyr, "Signal analysis and performance evaluation of a vehicle crash test with a fixed safety barrier based on Haar wavelets," International Journal of Wavelets, Multiresolution and Information Processing, vol. 9, no. 1, pp. 131-149, 2011.

[19] W. Pawlus, H. R. Karimi, and K. G. Robbersmyr, "A fuzzy logic approach to modeling a vehicle crash test," Central European Journal of Engineering, vol. 3, no. 1, pp. 67-79, 2013.

[20] A. R. Varkonyi-Koczy, A. Rovid, and P. Varlaki, "Intelligent methods for car deformation modeling and crash speed estimation," in Proceedings of the 1st Romanian-Hungarian Joint Symposium on Applied Computational Intelligence, Timisoara, Romania, May 2004.

[21] E. van der Laan, F. Veldpaus, B. de Jager, and M. Steinbuch, "LPV modeling of vehicle occupants," in Proceedings of the AVEC 9th International Symposium on Advanced Vehicle Control, Kobe, Japan, 2008.

[22] A. M. Elmarakbi and J. W. Zu, "Crash analysis and modeling of two vehicles in frontal collisions using two types of smart front-end structures: an analytical approach using IHBM," International Journal of Crashworthiness, vol. 11, no. 5, pp. 467483, 2006.

[23] G. Belforte, F. Dabbene, and P. Gay, "LPV approximation of distributed parameter systems in environmental modelling," Environmental Modelling and Software, vol. 20, no. 8, pp. 1063 1070, 2005.

[24] T. Soderstrom and P. Stoica, System Identification, Prentice Hall, Upper Saddle River, NJ, USA, 1989.

[25] L. Ljung, System Identification: Theory for the User, Prentice Hall, Englewood Cliffs, NJ, USA, 1987. 


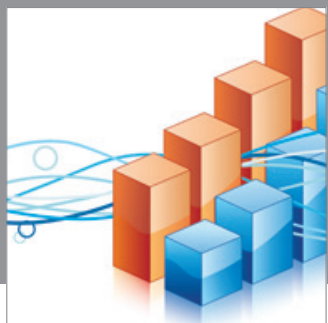

Advances in

Operations Research

mansans

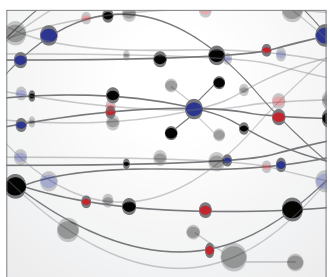

The Scientific World Journal
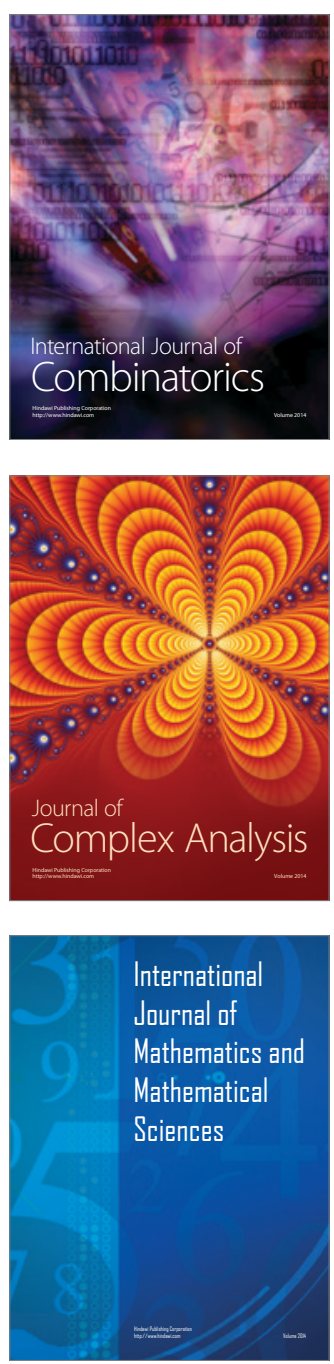
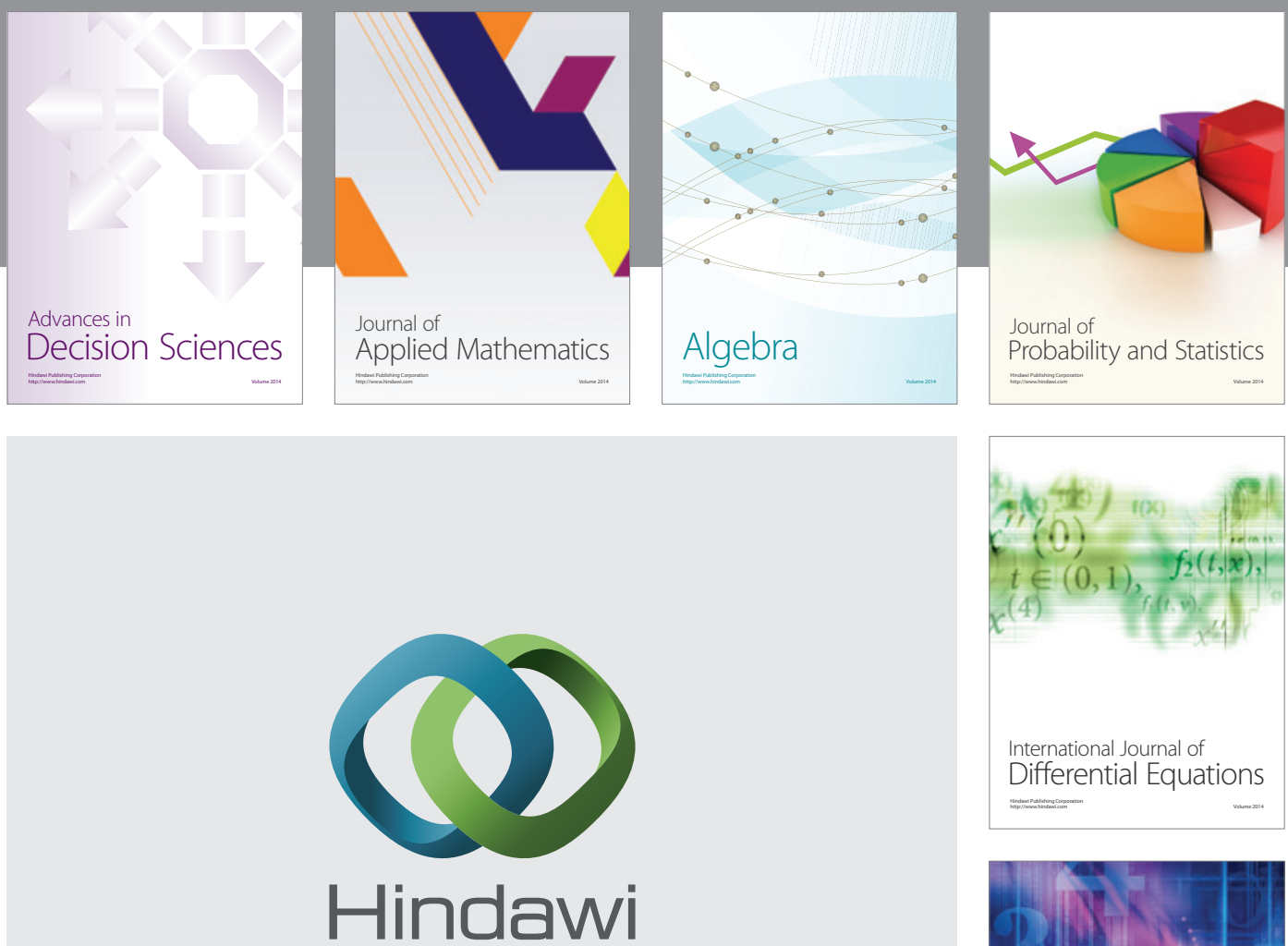

Submit your manuscripts at http://www.hindawi.com
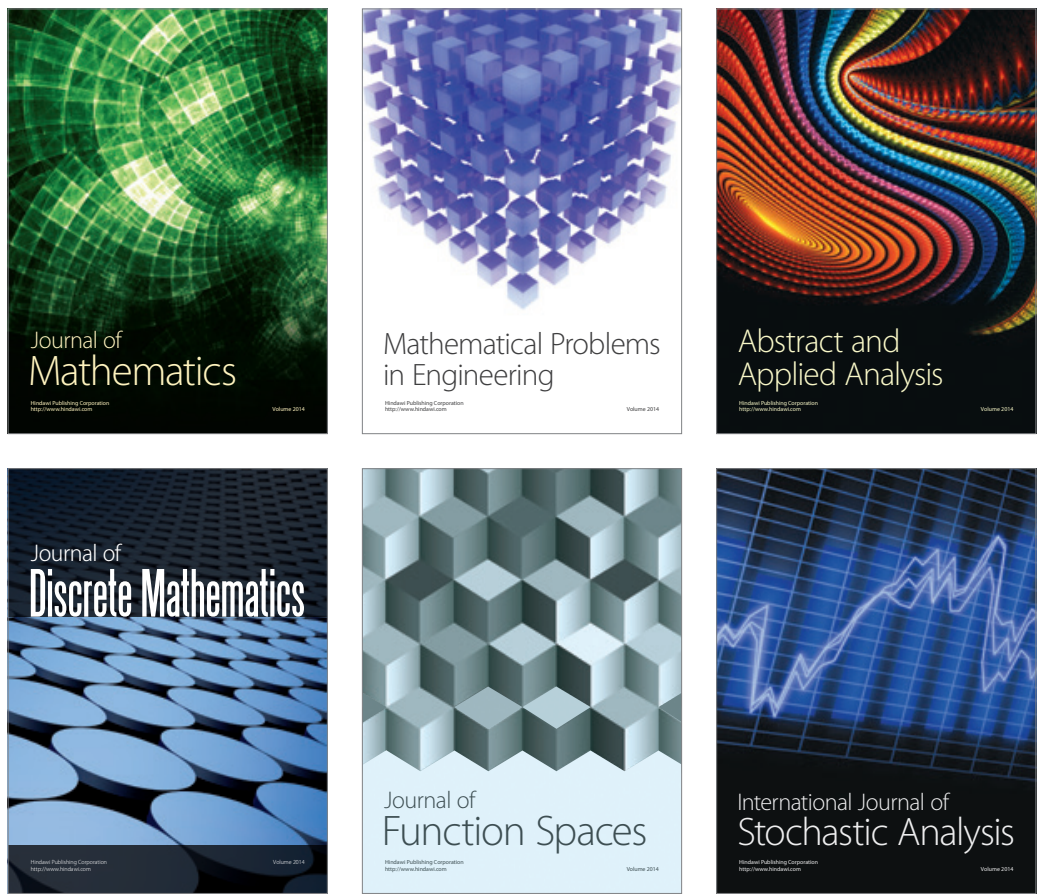

Journal of

Function Spaces

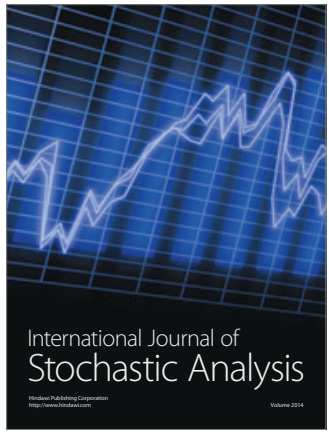

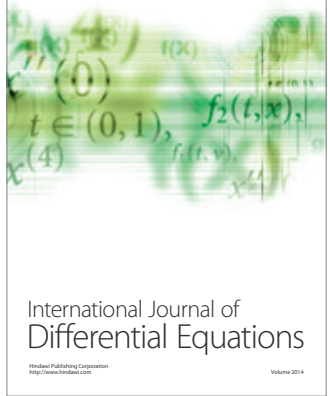
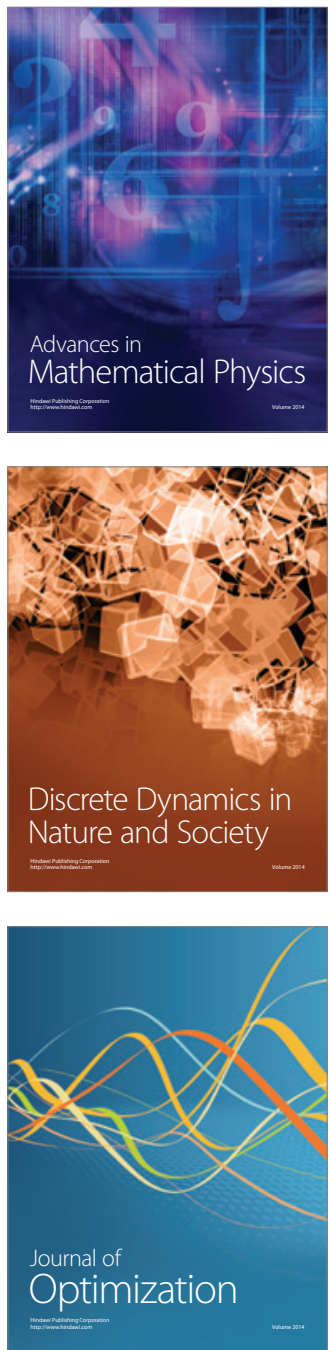